\title{
A prospective study of dietary patterns, meat intake and the risk of gestational diabetes mellitus
}

\author{
C. Zhang • M. B. Schulze • C. G. Solomon • F. B. Hu
}

Received: 27 April 2006 / Accepted: 19 July 2006 / Published online: 7 September 2006

(C) Springer-Verlag 2006

\begin{abstract}
Aims/hypothesis The aim of this study was to prospectively examine whether dietary patterns are related to risk of gestational diabetes mellitus (GDM).

Methods This prospective cohort study included 13,110 women who were free of cardiovascular disease, cancer, type 2 diabetes and history of GDM. Subjects completed a validated semi-quantitative food frequency questionnaire in 1991, and reported at least one singleton pregnancy between 1992 and 1998 in the Nurses' Health Study II. Two major dietary patterns (i.e. 'prudent' and 'Western') were identified through factor analysis. The prudent pattern
\end{abstract}

C. Zhang $(\bowtie) \cdot$ F. B. Hu

Department of Nutrition, Harvard School of Public Health, 665 Huntington Avenue,

Boston, MA 02115, USA

e-mail: cuilin.zhang@channing.harvard.edu

C. Zhang $\cdot$ F. B. Hu

Channing Laboratory, Department of Medicine,

Brigham and Women's Hospital and Harvard Medical School, Boston, MA, USA

M. B. Schulze

Department of Epidemiology,

German Institute of Human Nutrition,

Potsdam-Rehbruecke,

Nuthetal, Germany

C. G. Solomon

Divisions of General Medicine and Women's Health,

Department of Medicine,

Brigham and Women's Hospital and Harvard Medical School,

Boston, MA, USA

F. B. $\mathrm{Hu}$

Department of Epidemiology, Harvard School of Public Health,

Boston, MA, USA was characterised by a high intake of fruit, green leafy vegetables, poultry and fish, whereas the Western pattern was characterised by high intake of red meat, processed meat, refined grain products, sweets, French fries and pizza. Results We documented 758 incident cases of GDM. After adjustment for age, parity, pre-pregnancy BMI and other covariates, the relative risk (RR) of GDM, comparing the highest with the lowest quintile of the Western pattern scores, was 1.63 (95\% CI $\left.1.20-2.21 ; p_{\text {trend }}=0.001\right)$, whereas the RR comparing the lowest with the highest quintile of the prudent pattern scores was 1.39 (95\% CI 1.08-1.80; $\left.p_{\text {trend }}=0.018\right)$. The RR for each increment of one serving/ day was $1.61(95 \%$ CI $1.25-2.07)$ for red meat and 1.64 (95\% CI 1.13-2.38) for processed meat.

Conclusions/interpretation These findings suggest that prepregnancy dietary patterns may affect women's risk of developing GDM. A diet high in red and processed meat was associated with a significantly elevated risk.

Keywords Diet - Dietary pattern - Gestational diabetes .

Nutrition $\cdot$ Pregnancy $\cdot$ Red meat

\section{Abbreviations \\ FFQ food frequency questionnaire \\ GDM gestational diabetes mellitus \\ MET metabolic equivalent task \\ NHSII Nurses' Health Study II \\ $\mathrm{RR} \quad$ relative risk}

\section{Introduction}

Gestational diabetes mellitus (GDM) is among the most common complications of pregnancy in the United States; 
approximately 135,000 cases of GDM are diagnosed annually, representing an average $3-8 \%$ of all pregnancies [1]. Recent data have shown a substantial rise in the incidence of GDM among women of varied ethnic/racial backgrounds $[2,3]$. Women with GDM have an increased risk of prenatal morbidity and a considerably elevated risk of IGT and type 2 diabetes in the years following pregnancy [4-6]. Children of women with GDM are more likely to be obese [7] and to have IGT and diabetes in early adulthood [8].

Obesity is the major recognised modifiable risk factor for GDM. High pre-pregnancy BMI has been consistently associated with an increased risk of GDM [9, 10]. In the past decade, substantial evidence from epidemiological and clinical studies has suggested that diet influences glucose homeostasis and that modification of diet can influence the risk of type 2 diabetes among non-pregnant individuals. Recently, a 'Western' dietary pattern, characterised by a high intake of red meat, processed meat, sweets and refined grains, has been associated with an increased risk of type 2 diabetes in large prospective cohorts of both men and nonpregnant women [11, 12]. In addition, a conservative pattern similar to the Western pattern [13] in a Finnish population, a 'junk food' pattern in Native Canadians [14], and a modern western diet in Pima Indians [15] have all been reported to be associated with an increased risk of IGT and/or type 2 diabetes.

Limited attention has been given to the role of dietary patterns in the pathogenesis of GDM. Available data have predominantly focused on single nutrients. For example, significantly positive associations were observed between GDM risk and dietary intakes of total fat [16, 17] and saturated fat [18], while inverse associations were observed for polyunsaturated fat and GDM risk [18, 19] in some cross-sectional or retrospective studies. We were unaware of studies on the association of dietary pattern with the risk of GDM. Thus, we designed the present study to prospectively examine the association between major pregravid dietary patterns and GDM risk in a large cohort of US women.

\section{Subjects and methods}

\section{Study population}

The Nurses' Health Study II (NHSII), established in 1989, is a prospective cohort study of 116,671 female nurses in the US. This cohort has been, and continues to be, followed with the use of biennially mailed questionnaires to update information on health-related behaviours and characteristics and to determine incident disease outcomes. The follow-up rate has consistently exceeded $90 \%$ in every 2 -year period. For the analyses presented here, women were excluded if they did not complete a food frequency questionnaire (FFQ) in 1991, or if more than nine items on it were left blank; if their reported dietary intake was implausible with regard to total energy intake (i.e. $<2.09 \mathrm{MJ}[500 \mathrm{kcal}] /$ day or $>14.64 \mathrm{MJ}[3,500 \mathrm{kcal}] /$ day); or if they reported a multiple gestation (i.e. twins or higher order multiple gestations). Women were also excluded if they reported a history of diabetes, cancer, cardiovascular disease or GDM on the 1989 or 1991 questionnaire. The final sample for the current analyses consisted of 13,110 women who reported having at least one singleton pregnancy lasting 6 months or more between 1992 and 1998. The study was approved by the Human Research Committees at the Brigham and Women's Hospital; completion of the self-administered questionnaire was considered to imply informed consent.

\section{Dietary assessment}

Dietary intake information was collected using a 133-food item semi-quantitative FFQ designed to assess average food intake over the previous year. A standard portion size was given for each food item. Study participants were asked to choose from nine possible frequency responses, ranging from 'never' to 'more than six times a day', for each food. The selected frequency category for each food item was then converted to a daily intake. For example, a response of 'two to four servings per week' was converted to 0.43 servings per day (three servings per week). Nutrient intakes were computed by multiplying the frequency response by the nutrient content of the specified portion sizes. Nutritional values were derived from the US Department of Agriculture sources [20] and supplemented with information from manufacturers.

Food items with similar nutrient profiles or culinary uses were combined, and 39 predefined food groups were formed. This classification follows that of a study in men with a similar dietary assessment instrument [21]. A food item was classified individually if its composition differed substantially from that of other foods (for example, eggs or pizza), or if they were suspected to represent distinct dietary patterns (for example, wine or French fries). Questionnaire items on processed meat consumption included 'bacon', 'hot dogs', and 'sausage, salami, bologna and other processed meats', and items on red meat consumption included 'beef or lamb as main dish', 'pork as main dish', 'hamburger', and 'beef, pork or lamb as a sandwich or mixed dish'. Previous validation studies of FFQs similar to those used in the NHSII cohort showed good correlations between foods assessed by the FFQ and multiple weeks of food records completed over the previous year [22, 23]. For example, the corrected correlation coefficients between FFQ and multiple dietary records were 0.56 for hot dogs, 0.70 for bacon, 0.55 for sausage, salami, bologna, and other 
processed meats, 0.58 for poultry, and 0.66 for fish. The mean correlation coefficient between frequencies of intake of 55 foods from two FFQs administered 12 months apart was 0.57 [22].

Assessment of non-dietary covariates

Participants provided socio-demographic, clinical and lifestyle information biennially, including age, weight and smoking status. We calculated BMI as the ratio of weight in kilograms to squared height in metres. Self-reports of body weight were shown to be highly correlated with technicianmeasured weights $(r=0.96)$ [24]. Family history of diabetes was reported in 1989 only. Physical activity was assessed with the 1991 and 1997 questionnaires and was measured in weekly metabolic equivalent task hours (MET-h). Correlations between physical activity reported on recalls and diaries and that reported on the questionnaire in our cohort were 0.79 and 0.62 , respectively [25].

\section{Ascertainment of GDM}

The diagnosis of GDM was based on self-reported information in the biennial questionnaire. A previous validation study of GDM based on medical record review in this cohort demonstrated a high validity of self-reported diagnosis of GDM $[10,26]$. In brief, we reviewed the medical records of a sample of 114 women in the cohort who corroborated on a supplementary questionnaire that they had a first diagnosis of GDM in a singleton pregnancy between 1989 and 1991. Of these women, 94\% were confirmed to have been diagnosed with GDM by a physician on record review. All women reporting this diagnosis had evidence of abnormal glucose homeostasis. Formal National Diabetes Data Group criteria were used by most physicians for the diagnosis of GDM [26]. We also sent supplementary questionnaires to 100 women reporting a pregnancy uncomplicated by GDM during the same interval. Eighty-three per cent reported a glucose loading test, and all reported frequent urine screening in pregnancy, consistent with a high degree of surveillance in this cohort.

\section{Statistical analysis}

In the primary analysis, dietary patterns were generated by principal component factor analysis based on the 39 predefined food groups. This method identified dietary patterns primarily based on the correlations between these food groups. An orthogonal rotation procedure was used to maintain the uncorrelated nature of factors. The number of factors retained was determined by eigenvalue, the Scree test and the natural interpretability for each factor. The factor score for each pattern was calculated by summing intakes of food groups weighted by their factor loadings. Factor loadings represented correlation coefficients between the food groups and a particular pattern. The larger the loading of a given food item or group to the factor, the greater the contribution of that food item or group to a specific factor. Each woman received a factor score for each identified pattern. We then used dietary pattern scores to rank participants according to the degree to which they conformed to each dietary pattern. We divided dietary pattern scores into quintiles. In secondary analyses, we also constructed dietary pattern scores using only key food items for each pattern (i.e. red meat, processed meat, refined grain products, snacks, sweets and deserts, French fries and pizza for Western pattern; fruits, tomatoes, cabbages, green leafy vegetables, dark yellow vegetables, legumes, other vegetables, poultry and fish for prudent pattern) and omitting weights, as previously described in studies of the NHSII cohort [27] and the European Prospective Investigation into Cancer and Nutrition [28].

Relative risks (RRs) of GDM for each category of dietary pattern or food intake compared with the lowest category were estimated using Cox proportional hazards analysis [29] stratified according to 5-year age categories. The 1991 intake was used for the follow-up between 1991 and 1995, and the average of the 1991 and 1995 intake was used for the follow-up between 1995 and 1999 to reduce within-person variation [30]. Because women with previous GDM were excluded from this study, nulliparous women were over-represented in the GDM group. To account for this effect, we adjusted for parity in multivariate analyses and performed a secondary analysis restricted to nulliparous women $(n=5,039,266$ GDM cases $)$. In multivariate analyses, we additionally adjusted for age, BMI $(<21,21-$ $22.9,23.0-24.9,25.0-26.9,27.0-28.9,29.0-30.9,31.0-$ $32.9,33.0-34.9$ and $\geq 35.0 \mathrm{~kg} / \mathrm{m}^{2}$ ), smoking status (never/ past/current), race/ethnicity (Europid/African-American/ Hispanic/Asian), family history of diabetes, physical activity (quintiles), and dietary variables (quintile) including total fat (\% energy), cereal fibre, alcohol consumption, glycaemic load and total energy intake (quintile). We also conducted stratified analyses to assess whether the association between dietary pattern and GDM risk was modified by family history of diabetes (yes/no), adiposity status (BMI: $<30 \mathrm{vs} \geq 30 \mathrm{~kg} / \mathrm{m}^{2} ;<25 \mathrm{vs} \geq 25 \mathrm{~kg} / \mathrm{m}^{2}$ ) or physical activity status (high/low; divided by median).

When the association between GDM risk and intakes of red meat and processed meat was evaluated, total red and processed meat intakes were categorised into quintiles, while single meat item intakes were divided into three categories $(0$, $<0.14$ serving per day [one serving per week] and $\geq 0.14$ serving per day [one serving per week]), owing to the small number of participants with frequent intake. The significance of linear trends across categories of dietary intake was tested 
by assigning each participant the median value for the category and modelling this value as a continuous variable. All tests of statistical significance were two-sided. All statistical analyses were performed with SAS statistical software, version 8.12 (SAS institute, Cary, NC, USA).

\section{Results}

Two major dietary patterns were derived through factor analysis based on the 39 predefined food groups. The first factor was positively correlated with intakes of fruits, green leafy vegetables, poultry and fish, while the second factor was positively correlated with intakes of red meat, processed meat, refined grain products, sweets and deserts, French fries and pizza. The first factor explained $9.4 \%$ of the total variance and the second factor explained $5.9 \%$ of the total variance. As with our previous study [30], we labelled the first factor as the prudent pattern and the second factor as the Western pattern. Women with a high Western pattern score tended to smoke more, consume less fibre, and engage in less physical activity, whereas women with a high prudent pattern score tended to smoke less, consume more fibre, and to be more physically active (Table 1).

Over the 8 years of follow-up, 758 women reported a first diagnosis of GDM. After multivariate adjustment, the prudent pattern was significantly and inversely associated with GDM risk (Table 2). In contrast, a Western pattern score was significantly and positively associated with GDM risk. This association remained strong after adjustment for age, parity, pre-pregnancy BMI and other covariates. We next examined the joint effect of Western pattern and prudent pattern. After adjustment for age, parity, BMI, energy intake and other covariates, compared with women in the lowest quintile of the Western score but highest quintile of the prudent score, those in the highest quintile of the Western score and lowest quintile of the prudent score had a 1.86-fold (95\% CI 1.13-3.06) higher risk of GDM ( $p$ value for interaction 0.45 ). In stratified analysis, a positive association of the Western pattern with GDM risk persisted within strata defined by BMI, family history of diabetes and physical activity level ( $p$ values for interaction all >0.10). Additionally, we restricted our analysis to nulliparous women and obtained similar results. The association between a Western diet and GDM risk was no longer significant after further adjustment for red meat and processed meat; corresponding RRs for GDM across increasing quintiles of Western pattern scores were 1.00 (referent), 0.92 (95\% CI 0.71-1.19), 0.92 (95\% CI 0.69 $1.22), 0.86$ (95\% CI $0.62-1.18)$, and 1.03 (95\% CI 0.72 $1.48)\left(p_{\text {trend }}=0.697\right)$.

Intakes of red meat and processed meat were both significantly associated with a higher risk of GDM (Table 3).
To reduce the effect of other components of the Western pattern, we further controlled for the Western pattern score. The associations were slightly attenuated but generally remained strong: RRs across increasing quintiles of red meat were $1.00,1.25,1.51,1.70,1.65$ (95\% CI 1.24-2.18) $\left(p_{\text {trend }}=0.0007\right)$ and $1.00,1.28,1.31,1.52,1.55(95 \% \mathrm{CI}$ $1.17-2.06)\left(p_{\text {trend }}=0.017\right)$ for processed meat. Of note, the associations remained significant even after further controlling for other dietary factors, including glycaemic load, cereal fibre and magnesium.

To investigate major contributors to the observed associations between red and processed meat intake and GDM risk, we adjusted for those major nutrients in red and processed meat that have been associated with glucose intolerance. The associations with GDM remained significant for both red meat and processed meat after adjustment for fatty acids and cholesterol: RRs across increasing quintiles of red meat were $1.00,1.18,1.39,1.59,1.55$ (95\% CI 1.13-2.12) $\left(p_{\text {trend }}=0.006\right)$ and $1.00,1.21,1.19,1.38,1.41$ (95\% CI 1.07-1.86) $\left(p_{\text {trend }}=0.049\right)$ for processed meat. After the adjustment for dietary haem iron, the association between processed meat and GDM was attenuated, but remained significant $\left(p_{\text {trend }}=0.01\right)$, whereas the association of red meat with GDM risk became insignificant $\left(p_{\text {trend }}=0.08\right)$. When red and processed meat products were analysed simultaneously as continuous variables in multivariate models, we observed a RR of 1.61 (95\% CI 1.25-2.07) for red meat and a RR of 1.64 (95\% CI 1.13-2.38) for processed meats for each serving increment in consumption. Among specific processed meat, intakes of sausage, salami, bologna and other processed meats showed the strongest positive association.

To avoid potential bias as a result of undiagnosed nongestational diabetes, we eliminated GDM cases who reported non-gestational diabetes in the next round questionnaire $(n=20)$; the results did not change materially. Similar results were observed when BMI was fitted as a continuous variable in multivariate models.

Moreover, findings from secondary analysis using simplified dietary pattern scores, based on only key food items without weights, were broadly consistent with those for pattern variables based on all food items and using weights. The simplified pattern variables showed a correlation $>0.92$ with the more comprehensive scores derived from the principal component factor analysis for both the Western pattern and the prudent pattern.

\section{Discussion}

In this large prospective study we observed strong associations of the Western and prudent dietary patterns with GDM risk. The association with the Western pattern was largely explained by intakes of red and processed meat 
Table 1 Baseline (1991) characteristics according to quintile of dietary pattern scores

\begin{tabular}{|c|c|c|c|c|c|c|}
\hline \multirow[t]{2}{*}{ Characteristics } & \multicolumn{3}{|c|}{ Western } & \multicolumn{3}{|c|}{ Prudent } \\
\hline & Q1 & Q3 & Q5 & Q1 & Q3 & Q5 \\
\hline$n$ & 2,666 & 2,639 & 2,530 & 2,669 & 2,608 & 2,519 \\
\hline Age, years & 31.9 & 31.5 & 31.1 & 30.7 & 31.6 & 32.2 \\
\hline BMI $\left(\mathrm{kg} / \mathrm{m}^{2}\right)$ & 22.9 & 23.4 & 24.1 & 23.4 & 23.4 & 23.5 \\
\hline Physical activity (MET-h/week) ${ }^{*}$ & 28.5 & 21.8 & 19.6 & 16.3 & 20.9 & 33.2 \\
\hline Current smoking (\%) & 7.0 & 7.7 & 12.0 & 12.7 & 7.4 & 7.6 \\
\hline Nulliparous (\%) & 55.1 & 36.1 & 30.5 & 40.6 & 38.6 & 40.6 \\
\hline White $(\%)$ & 93.1 & 94.3 & 95.1 & 93.7 & 95.0 & 94.6 \\
\hline Family history of diabetes (\%) & 12.6 & 12.0 & 12.8 & 11.8 & 12.5 & 12.3 \\
\hline Alcohol consumption (g/day) & 2.7 & 3.0 & 3.3 & 2.3 & 3.1 & 3.7 \\
\hline \multicolumn{7}{|l|}{ Total energy } \\
\hline $\mathrm{MJ} /$ day & 5.64 & 7.49 & 10.18 & 6.55 & 7.53 & 9.17 \\
\hline $\mathrm{kcal} /$ day & 1,349 & 1,790 & 2,432 & 1,565 & 1,799 & 2,191 \\
\hline Carbohydrate (\% energy) & 53.0 & 50.4 & 48.8 & 49.0 & 50.5 & 53.1 \\
\hline Protein (\% energy) & 20.5 & 19.2 & 17.9 & 18.0 & 19.4 & 19.9 \\
\hline Total fat ( $\%$ energy) & 27.4 & 31.1 & 34.0 & 33.6 & 30.8 & 28.2 \\
\hline trans-Fat ( $\%$ energy) & 1.3 & 1.6 & 1.9 & 2.0 & 1.5 & 1.2 \\
\hline Polyunsaturated fat (\% energy) & 5.1 & 5.4 & 5.7 & 5.3 & 5.4 & 5.5 \\
\hline Monounsaturated fat ( $\%$ energy) & 10.1 & 11.7 & 13.1 & 12.9 & 11.5 & 10.5 \\
\hline Saturated fat (\% energy) & 9.7 & 11.3 & 12.4 & 12.5 & 11.2 & 9.7 \\
\hline \multicolumn{7}{|l|}{ Nutrient intake (energy-adjusted) } \\
\hline Total fibre (g/day) & 21.0 & 17.6 & 15.9 & 13.8 & 17.8 & 23.0 \\
\hline Cereal fibre (g/day) & 6.9 & 5.6 & 4.9 & 4.9 & 5.9 & 6.3 \\
\hline Fruit fibre (g/day) & 4.7 & 3.1 & 2.3 & 2.0 & 3.3 & 4.8 \\
\hline Vegetable fibre (g/day) & 7.8 & 6.3 & 5.4 & 4.0 & 6.2 & 9.7 \\
\hline Magnesium (mg/day) & 359.6 & 314.2 & 283.6 & 264.4 & 320.2 & 371.0 \\
\hline Caffeine (mg/day) & 193.3 & 182.0 & 190.1 & 192.0 & 182.9 & 193.0 \\
\hline Total iron (mg/day) & 34.1 & 31.5 & 26.1 & 26.8 & 31.9 & 31.2 \\
\hline Haem Iron (mg/day) & 0.4 & 1.1 & 1.1 & 1.1 & 1.1 & 1.0 \\
\hline Glycaemic load (energy-adjusted) & 184.1 & 176.1 & 169.0 & 178.1 & 175.2 & 176.9 \\
\hline Glycaemic index (energy-adjusted) & 75.3 & 77.3 & 78.3 & 79.5 & 76.8 & 74.7 \\
\hline \multicolumn{7}{|l|}{ Food intake (servings/day) } \\
\hline Fish & 0.27 & 0.27 & 0.27 & 0.15 & 0.25 & 0.43 \\
\hline Poultry & 0.43 & 0.46 & 0.48 & 0.30 & 0.45 & 0.62 \\
\hline Red meat & 0.24 & 0.50 & 0.88 & 0.61 & 0.52 & 0.45 \\
\hline Processed meat & 0.07 & 0.19 & 0.47 & 0.28 & 0.23 & 0.17 \\
\hline Fruit & 1.3 & 1.2 & 1.2 & 0.5 & 1.1 & 2.2 \\
\hline Vegetables & 3.2 & 3.1 & 3.3 & 1.4 & 2.9 & 5.9 \\
\hline Green leafy vegetables & 0.7 & 0.6 & 0.6 & 0.26 & 0.57 & 1.22 \\
\hline Whole grains & 1.0 & 1.1 & 1.2 & 0.54 & 1.07 & 1.82 \\
\hline Refined grains & 0.95 & 1.36 & 2.12 & 1.31 & 1.43 & 1.61 \\
\hline Low-fat dairy & 1.56 & 1.63 & 1.53 & 1.01 & 1.68 & 2.04 \\
\hline High-fat dairy & 0.55 & 0.98 & 1.52 & 0.93 & 1.00 & 1.04 \\
\hline Eggs & 0.09 & 0.17 & 0.29 & 0.15 & 0.18 & 0.21 \\
\hline Desserts and sweets & 0.47 & 0.92 & 1.65 & 1.10 & 0.97 & 0.87 \\
\hline Fries & 0.04 & 0.09 & 0.23 & 0.17 & 0.10 & 0.07 \\
\hline Pizza & 0.08 & 0.12 & 0.19 & 0.14 & 0.12 & 0.11 \\
\hline
\end{tabular}

All data are presented as mean values unless otherwise specified

Q1 is the lowest quintile, Q3 is the medium quintile, and Q5 is the highest quintile

*MET-h/week, calculated using the duration per week of various forms of exercise, weighting each activity by its intensity level

products. Pregravid intake of red and processed meats were both significantly and positively associated with GDM risk, independently of known risk factors for type 2 diabetes and GDM, including BMI, physical activity and other compo- nents of the Western pattern (i.e. refined grain products, snacks, sweets and deserts, French fries and pizza).

Limited attention has been given to the role of dietary patterns in the aetiology of GDM, with available data 
Table 2 Relative risks of GDM (with 95\% CIs) according to quintiles of pre-pregnancy dietary pattern scores

\begin{tabular}{|c|c|c|c|c|c|c|}
\hline & \multicolumn{5}{|l|}{ Quintiles } & \multirow[t]{2}{*}{$p_{\text {trend }}$} \\
\hline & 1 & 2 & 3 & 4 & 5 & \\
\hline \multicolumn{7}{|c|}{ Western pattern score } \\
\hline Number of cases & 127 & 135 & 151 & 155 & 190 & \\
\hline Person-years & 19,231 & 20,227 & 20,269 & 20,146 & 19,759 & \\
\hline RR1 & 1.00 & $1.16(0.91-1.40)$ & $1.39(1.09-1.76)$ & $1.49(1.18-1.89)$ & $1.97(1.57-2.48)$ & $<0.0001$ \\
\hline RR2 & 1.00 & $1.11(0.87-1.42)$ & $1.28(1.01-1.62)$ & $1.34(1.06-1.70)$ & $1.68(1.33-2.11)$ & $<0.0001$ \\
\hline RR3 & 1.00 & $1.09(0.85-1.41)$ & $1.22(0.94-1.59)$ & $1.25(0.94-1.65)$ & $1.63(1.20-2.21)$ & 0.0011 \\
\hline \multicolumn{7}{|c|}{ Prudent pattern score } \\
\hline Number of cases & 177 & 151 & 138 & 163 & 129 & \\
\hline Person-years & 19,901 & 20,066 & 20,000 & 20,572 & 19,093 & 0.010 \\
\hline RR1 & $1.41(1.12-1.77)$ & $1.18(0.94-1.50)$ & $1.07(0.84-1.36)$ & $1.21(0.96-1.53)$ & 1.00 & 0.017 \\
\hline RR2 & $1.37(1.09-1.72)$ & $1.19(0.94-1.51)$ & $1.07(0.84-1.36)$ & $1.20(0.95-1.51)$ & 1.00 & 0.018 \\
\hline
\end{tabular}

RR1 adjusted for age (5-year category) and parity $(0,1,2,3+)$

RR2 adjusted for age (5-year category), parity (0, 1, 2, 3+), BMI (nine categories: $<21,21-22.9,23.0-24.9,25.0-26.9,27.0-28.9,29.0-30.9$, $31.0-32.9,33.0-34.9$ and $\left.\geq 35.0 \mathrm{~kg} / \mathrm{m}^{2}\right)$

RR3 adjusted for age (5-year category), parity $(0,1,2,3+)$, BMI (nine categories), race/ethnicity, cigarette smoking status (never, past, current), family history of diabetes in a first-degree relative (yes, no), alcohol intake ( $0,0.1-5.0,5.1-15.0$ or $>15 \mathrm{~g} /$ day), physical activity (quintile) and total energy (quintile)

predominantly focusing on the association of single nutrients with GDM risk. Our findings are in general accordance with evidence of positive associations of a Western pattern diet $[11,12]$, dietary patterns similar to the Western pattern [13-15], and red meat intakes [11, 27, 31, $32]$ with type 2 diabetes in men and non-pregnant women. The observed positive associations of pregravid dietary patterns and meat intakes with GDM risk are biologically plausible. Normal pregnancy, especially the third trimester, is characterised by profound metabolic stresses on maternal lipid and glucose homeostasis, including marked insulin resistance and hyperinsulinaemia [33]. Women who develop GDM are thought to have a compromised capacity to adapt to the increased insulin resistance characteristic of late pregnancy (mainly third trimester) [33]. Pregnancyrelated metabolic challenges unmask a predisposition to glucose metabolic disorders in some women. The majority of women with GDM have beta cell dysfunction on a background of chronic insulin resistance to which the insulin resistance of pregnancy is partially additive [34]. Factors that contribute to insulin resistance before pregnancy may have a deleterious effect during pregnancy and be risk factors for GDM. The Western pattern score was significantly associated with fasting insulin and C-peptide levels in US male health professionals [35]. Moreover, a dietary pattern that resembled the Western pattern was associated with higher plasma glucose concentrations in a Dutch population [36].

A major characteristic of the Western pattern is a high intake of processed and red meat. In the present study, the association between a Western diet and GDM risk became non-significant after adjustment for red meat and processed meat, suggesting that the observed association may be largely due to intakes of these foods. Several possible biologically adverse effects of components in red and processed meats, such as saturated fatty acids and cholesterol, on insulin sensitivity have been proposed and might be relevant to the pathophysiology of GDM. In the present study, the strong association of red meat and processed meat with GDM risk remained significant after further adjustment for other dietary factors, including fatty acids and cholesterol, indicating that components of red meat and processed meat other than these nutrients might be also relevant to the pathogenesis of GDM. For example, nitrites, frequently used as a preservative in processed meats, have been implicated in the development of diabetes. Nitrosamines can be formed by the interaction of amino compounds with nitrites present either in the stomach or within the food product [37]. They have been linked to beta cell toxicity [38]. In addition, low doses of the nitrosamine streptozotocin were found to induce type 2 diabetes in animal models [39].

Another potential explanation is related to the toxic effects of AGEs, which can be formed in meat and high-fat products through heating and processing [40]. Animal models and human studies suggest that AGEs are involved in the progression of diabetes. The development of type 2 diabetes was reduced by treatment with aminoguanidine, an AGE inhibitor, in genetically diabetic mice [41], and improvement of various features of insulin resistance was shown in mice fed a diet low in AGEs [42]. Moreover, a diet high in AGEs was found to promote inflammatory mediators that might be important in the genesis of diabetes, such as TNF- $\alpha$ and C-reactive protein [43]. Both 
Table 3 Relative risks of GDM (with 95\% CIs) according to quintiles of intakes of subtypes of red meat

\begin{tabular}{|c|c|c|c|c|c|c|}
\hline & \multicolumn{5}{|c|}{ Quintiles } & \multirow{2}{*}{$p_{\text {trend }}$} \\
\hline & 1 & 2 & 3 & 4 & 5 & \\
\hline \multicolumn{7}{|c|}{ Red meat (servings/day) } \\
\hline Range & $0-0.21$ & $0.28-0.35$ & $0.42-0.56$ & $0.57-0.85$ & $0.86-3.50$ & \\
\hline Median & 0.14 & 0.35 & 0.49 & 0.71 & 1.07 & \\
\hline Number of cases & 118 & 135 & 173 & 152 & 180 & \\
\hline Person-years & 21,965 & 20,925 & 21,955 & 16,691 & 18,366 & \\
\hline RR1 & 1.00 & $1.37(1.07-1.76)$ & $1.78(1.40-2.25)$ & $2.16(1.69-2.75)$ & $2.36(1.86,2.99)$ & $<0.0001$ \\
\hline RR2 & 1.00 & $1.28(1.00-1.64)$ & $1.59(1.26-2.02)$ & $1.87(1.46-2.38)$ & $1.92(1.52,2.44)$ & $<0.0001$ \\
\hline RR3 & 1.00 & $1.25(0.97-1.60)$ & $1.52(1.19-1.94)$ & $1.73(1.35-2.23)$ & $1.74(1.35,2.26)$ & $<0.0001$ \\
\hline \multicolumn{7}{|c|}{ Total processed meat (servings/day) } \\
\hline Range & 0 & 0.07 & 0.14 & $0.21-0.35$ & $0.42-4.78$ & \\
\hline Median & 0 & 0.07 & 0.14 & 0.21 & 0.57 & \\
\hline Number of cases & 104 & 127 & 147 & 185 & 195 & \\
\hline Person-years & 18,411 & 18,136 & 21,341 & 21,022 & 20,722 & \\
\hline RR1 & 1.00 & $1.41(1.09-1.83)$ & $1.55(1.20-1.99)$ & $1.90(1.49-2.42)$ & $2.21(1.73,2.81)$ & $<0.0001$ \\
\hline RR2 & 1.00 & $1.33(1.03-1.73)$ & $1.40(1.08 .1 .80)$ & $1.68(1.31-2.14)$ & $1.87(1.46,2.38)$ & $<0.0001$ \\
\hline RR3 & 1.00 & $1.29(0.99-1.67)$ & $1.33(1.03-1.72)$ & $1.58(1.23-2.02)$ & $1.68(1.30,2.16)$ & 0.0003 \\
\hline \multicolumn{7}{|l|}{ Bacon } \\
\hline Range & & 0 & 0.07 & $\geq 0.14$ & & \\
\hline Number of cases & & 328 & 333 & 97 & & \\
\hline Person-years & & 48,102 & 41,701 & 9,829 & & \\
\hline RR1 & & 1.00 & $1.43(1.23-1.67)$ & $1.51(1.20-1.89)$ & & $<0.0001$ \\
\hline RR2 & & 1.00 & $1.35(1.16-1.58)$ & $1.37(1.10-1.73)$ & & 0.0002 \\
\hline RR3 & & 1.00 & $1.32(1.13-1.55)$ & $1.29(1.02-1.63)$ & & 0.002 \\
\hline \multicolumn{7}{|l|}{ Hot dogs } \\
\hline Range & & 0 & 0.07 & $\geq 0.14$ & & \\
\hline Number of cases & & 281 & 355 & 122 & & \\
\hline Person-years & & 37,770 & 47,685 & 14,177 & & \\
\hline RR1 & & 1.00 & $1.41(1.20-1.66)$ & $1.60(1.29-1.99)$ & & $<0.0001$ \\
\hline RR2 & & 1.00 & $1.29(1.10-1.52)$ & $1.38(1.11-1.72)$ & & 0.0007 \\
\hline RR3 & & 1.00 & $1.22(1.04-1.44)$ & $1.25(1.00-1.56)$ & & 0.02 \\
\hline \multicolumn{7}{|c|}{ Sausage, salami, bologna and other processed meats } \\
\hline Range & & 0 & 0.07 & $\geq 0.14$ & & \\
\hline Number of cases & & 183 & 309 & 266 & & \\
\hline Person-years & & 28,484 & 44,538 & 26,610 & & \\
\hline RR1 & & 1.00 & $1.40(1.16-1.68)$ & $1.92(1.59-2.32)$ & & $<0.0001$ \\
\hline RR2 & & 1.00 & $1.30(1.08-1.57)$ & $1.72(1.42-2.08)$ & & $<0.0001$ \\
\hline RR3 & & 1.00 & $1.26(1.05-1.52)$ & $1.60(1.31-1.95)$ & & $<0.0001$ \\
\hline
\end{tabular}

RR1 adjusted for age (5-year category) and parity $(0,1,2,3+)$

RR2 adjusted for age (5-year category), parity (0, 1, 2, 3+), and BMI (nine categories: <21, 21-22.9, 23.0-24.9, 25.0-26.9, 27.0-28.9, 29.0-30.9, $31.0-32.9,33.0-34.9$, and $\geq 35.0 \mathrm{~kg} / \mathrm{m}^{2}$ )

RR3 adjusted for age (5-year category), parity $(0,1,2,3+)$, BMI (nine categories), race/ethnicity, cigarette smoking status (never, past, current), family history of diabetes in a first-degree relative (yes, no), alcohol intake (0,0.1-5.0, 5.1-15.0 or $>15 \mathrm{~g} /$ day), physical activity (quintile), and total energy (quintile)

biomarkers have been positively associated with the risk of GDM or gestational hyperglycaemia [44, 45]. In addition, it has been proposed that food-derived AGEs may interact with glycoxidation-related genes, in particular, the AGE receptor $(A G E R)$ gene, to determine risk of type 2 diabetes by activating the AGE receptor signal transduction pathway involved in the inflammatory response [46]. Haem iron in red meat might also contribute to the increased risk of GDM, because body iron overload has been postulated to promote insulin resistance and increase the risk of type 2 diabetes [47]. More recently, iron supplements and increased iron stores in pregnant women without iron deficiency were related to an increased risk of GDM [48]. However, the association between processed meat and GDM risk remained strong after adjusting for haem iron. Nevertheless, it is also plausible that other unidentified components in red meat and processed meat can contribute to the adverse effect on GDM related to them. 
Dietary information during pregnancy was not specifically measured in the current study. However, limited data suggest that dietary patterns and macronutrient intakes do not vary significantly between periods prior to conception and periods of pregnancy [49], although women generally increase their energy intake in pregnancy to meet fetal need. It is plausible that much of the adverse effects of red meat and processed meat on the risk of GDM that we observed for the pregravid period reflected continued consumption patterns during pregnancy. The current study addresses the relationship between diet and GDM risk only. Reduced intake of red meat may increase the risk of iron deficiency anaemia, which has been associated with other pregnancy complications, including an increased risk of preterm delivery [50]. Further studies are needed to examine relationships between dietary patterns in pregnancy and various pregnancy outcomes, including fetal growth and development.

Our validation study indicated a very high degree of surveillance during pregnancy in this cohort and a high degree of accuracy of self-reported GDM compared with medical record review [10, 26]. Misclassification of noncases as cases would be expected to attenuate observed associations between risk factors and outcome, and so would not explain positive results. Misclassification of dietary exposure is inevitable. However, because of the prospective design of this study, dietary exposure data could not have been influenced by the subsequent occurrence or non-occurrence of GDM. The misclassification would have weakened rather than strengthened the observed associations. Previous validation studies of FFQ similar to those used in the NHSII cohort showed good correlations between foods assessed by the FFQ and four 1week weighed diet records [22]. Limited variation of intakes for single red meat or processed meat items in our cohort could lead insufficient statistical power to detect significant associations. Because of the observational nature of the current study, we cannot rule out the possibility of residual confounding by unmeasured factors. However, significant associations remained after carefully controlling for major well-documented risk factors for GDM.

Because red and processed meats are components of the Western pattern, adjustment for the Western pattern in the analysis of association of red meat and processed meat with GDM risk might represent an over-adjustment. However, the main purpose of adjustment for the Western pattern in our analysis was to control for potential confounding by overall dietary patterns and other food items associated with red meat intake. Because risk estimates were only very moderately attenuated by adjustment for the Western pattern, it is unlikely that confounding by other food groups associated with red and processed meat intake explains our findings.
An advantage of using dietary patterns is the potential to detect the combined effect of foods, especially if the individual components of a pattern contribute to only a small amount of risk. Good reproducibility over time of the patterns generated by this method has been demonstrated in a parallel cohort of men [30]. Dietary patterns were consistent over time in our cohort, and our use of cumulative averages of dietary pattern scores reduced the influence of random error. The principal components method of pattern analysis identified existing patterns; therefore, the Western pattern observed in our study does not necessarily represent food choices that would pose the highest diabetes risk, nor does the prudent pattern represent the lowest risk. Although no other studies have examined the association between these dietary patterns and GDM risk, these and similar dietary patterns have been shown to be associated with type 2 diabetes among non-pregnant women and men [11-15]. In addition, we constructed less population-dependent pattern variables using simplified variables [28] and applied them in the analysis of the association between dietary patterns and GDM risk. Pattern scores derived from these two approaches were highly correlated, and findings on their association with GDM were generally consistent. These simplified food variables can be adopted in future studies in other populations for replicating findings from the present study.

In summary, although the observational design of our study makes it impossible to assess causation, our findings support the hypothesis that pregravid diet affects women's susceptibility to GDM when facing the profound metabolic challenges of late pregnancy. Independently of conventional risk factors for GDM and type 2 diabetes, the Western dietary pattern, in particular, higher red and processed meat intake, was strongly associated with a higher risk of GDM.

Acknowledgements This study was funded by research grant CA50385 and DK58845 from the National Institutes of Health. We are grateful to W. C. Willett and R. M. van Dam for their suggestions and comments.

Duality of interest There is no duality of interest to declare.

\section{References}

1. Metzger BE, Coustan DR (1998) Summary and recommendations of the 4th International Workshop-Conference on Gestational Diabetes Mellitus. The Organizing Committee. Diabetes Care 21 (Suppl 2):B161-B167

2. Ferrara A, Kahn HS, Quesenberry CP, Riley C, Hedderson MM (2004) An increase in the incidence of gestational diabetes mellitus: Northern California, 1991-2000. Obstet Gynecol 103:526-533

3. Dabelea D, Snell-Bergeon JK, Hartsfield CL, Bischoff KJ, Hamman RF, McDuffie RS (2005) Increasing prevalence of gestational diabetes mellitus (GDM) over time and by birth 
cohort: Kaiser Permanente of Colorado GDM Screening Program. Diabetes Care 28:579-584

4. American Diabetes Association (2004) Gestational diabetes mellitus. Diabetes Care 27(Suppl 1):S88-S90

5. Hanna FW, Peters JR (2002) Screening for gestational diabetes: past, present and future. Diabet Med 19:351-358

6. Kim C, Newton KM, Knopp RH (2002) Gestational diabetes and the incidence of type 2 diabetes: a systematic review. Diabetes Care 25:1862-1868

7. Boney CM, Verma A, Tucker R, Vohr BR (2005) Metabolic syndrome in childhood: association with birth weight, maternal obesity, and gestational diabetes mellitus. Pediatrics 115: e290-e296

8. Silverman BL, Metzger BE, Cho NH, Loeb CA (1995) Impaired glucose tolerance in adolescent offspring of diabetic mothers. Relationship to fetal hyperinsulinism. Diabetes Care 18:611-617

9. Berkowitz GS, Lapinski RH, Wein R, Lee D (1992) Race/ ethnicity and other risk factors for gestational diabetes. Am J Epidemiol 135:965-973

10. Solomon CG, Willett WC, Carey VJ et al (1997) A prospective study of pregravid determinants of gestational diabetes mellitus. JAMA 278:1078-1083

11. Fung TT, Schulze M, Manson JE, Willett WC, Hu FB (2004) Dietary patterns, meat intake, and the risk of type 2 diabetes in women. Arch Intern Med 164:2235-2240

12. van Dam RM, Rimm EB, Willett WC, Stampfer MJ, Hu FB (2002) Dietary patterns and risk for type 2 diabetes mellitus in US men. Ann Intern Med 136:201-209

13. Montonen J, Knekt P, Harkanen T et al (2005) Dietary patterns and the incidence of type 2 diabetes. Am J Epidemiol 161: 219-227

14. Gittelsohn J, Wolever TM, Harris SB et al (1998) Specific patterns of food consumption and preparation are associated with diabetes and obesity in a Native Canadian community. J Nutr 128:541-547

15. Swinburn BA, Boyce VL, Bergman RN, Howard BV, Bogardus C (1991) Deterioration in carbohydrate metabolism and lipoprotein changes induced by modern, high fat diet in Pima Indians and Caucasians. J Clin Endocrinol Metab 73:156-165

16. Moses RG, Shand JL, Tapsell LC (1997) The recurrence of gestational diabetes: could dietary differences in fat intake be an explanation? Diabetes Care 20:1647-1650

17. Wijendran V, Bendel RB, Couch SC et al (1999) Maternal plasma phospholipid polyunsaturated fatty acids in pregnancy with and without gestational diabetes mellitus: relations with maternal factors. Am J Clin Nutr 70:53-61

18. Bo S, Menato G, Lezo A et al (2001) Dietary fat and gestational hyperglycaemia. Diabetologia. 44:972-978

19. Wang Y, Storlien LH, Jenkins AB et al (2000) Dietary variables and glucose tolerance in pregnancy. Diabetes Care 23:460-464

20. USDA (1993) USDA national nutrient database for standard reference, release 10: nutrient data laboratory home page. Current information available from http://www.ars.usda.gov/ba/bhnrc/ndl last accessed in July 2006

21. Hu FB, Rimm EB, Stampfer MJ, Ascherio A, Spiegelman D, Willett WC (2000) Prospective study of major dietary patterns and risk of coronary heart disease in men. Am J Clin Nutr 72:912-921

22. Salvini S, Hunter DJ, Sampson L et al (1989) Food-based validation of a dietary questionnaire: the effects of week-toweek variation in food consumption. Int $\mathrm{J}$ Epidemiol 18: 858-867

23. Willett W (1998) Nutritional epidemiology, 2nd edn. Oxford University Press, New York
24. Willett W, Stampfer MJ, Bain C et al (1983) Cigarette smoking, relative weight, and menopause. Am J Epidemiol 117:651-658

25. Wolf AM, Hunter DJ, Colditz GA et al (1994) Reproducibility and validity of a self-administered physical activity questionnaire. Int J Epidemiol 23:991-999

26. Solomon CG, Willett WC, Rich-Edwards J et al (1996) Variability in diagnostic evaluation and criteria for gestational diabetes. Diabetes Care 19:12-16

27. Schulze MB, Manson JE, Willett WC, Hu FB (2003) Processed meat intake and incidence of Type 2 diabetes in younger and middle-aged women. Diabetologia 46:1465-1473

28. Schulze MB, Hoffmann K, Kroke A, Boeing H (2003) An approach to construct simplified measures of dietary patterns from exploratory factor analysis. Br J Nutr 89:409-419

29. Cox DR, Oakes D (1984) Analysis of survival data. Chapman and Hall, London

30. Hu FB, Rimm E, Smith-Warner SA et al (1999) Reproducibility and validity of dietary patterns assessed with a food-frequency questionnaire. Am J Clin Nutr 69:243-249

31. van Dam RM, Willett WC, Rimm EB, Stampfer MJ, Hu FB (2002) Dietary fat and meat intake in relation to risk of type 2 diabetes in men. Diabetes Care 25:417-424

32. Song Y, Manson JE, Buring JE, Liu S (2004) A prospective study of red meat consumption and type 2 diabetes in middle-aged and elderly women: The Women's Health Study. Diabetes Care 27:2108-2115

33. Knopp RH, Bergelin RO, Wahl PW, Walden CE, Chapman M, Irvine S (1982) Population-based lipoprotein lipid reference values for pregnant women compared to nonpregnant women classified by sex hormone usage. Am J Obstet Gynecol 143:626637

34. Buchanan TA, Xiang AH (2005) Gestational diabetes mellitus. J Clin Invest 115:485-491

35. Fung TT, Rimm EB, Spiegelman D et al (2001) Association between dietary patterns and plasma biomarkers of obesity and cardiovascular disease risk. Am J Clin Nutr 73:61-67

36. van Dam RM, Grievink L, Ocke MC, Feskens EJ (2003) Patterns of food consumption and risk factors for cardiovascular disease in the general Dutch population. Am J Clin Nutr 77:1156-1163

37. Lijinsky W (1999) $N$-Nitroso compounds in the diet. Mutat Res 443:129-138

38. Dahlquist G (1998) The aetiology of type 1 diabetes: an epidemiological perspective. Acta Paediatr Suppl 425:5-10

39. Ito M, Kondo Y, Nakatani A, Naruse A (1999) New model of progressive non-insulin-dependent diabetes mellitus in mice induced by streptozotocin. Biol Pharm Bull 22:988-989

40. Peppa M, Goldberg T, Cai W, Rayfield E, Vlassara H (2002) Glycotoxins: a missing link in the "relationship of dietary fat and meat intake in relation to risk of type 2 diabetes in men". Diabetes Care 25:1898-1899

41. Piercy V, Toseland CD, Turner NC (1998) Potential benefit of inhibitors of advanced glycation end products in the progression of type II diabetes: a study with aminoguanidine in C57/BLKsJ diabetic mice. Metabolism 47:1477-1480

42. Hofmann SM, Dong HJ, Li Z et al (2002) Improved insulin sensitivity is associated with restricted intake of dietary glycoxidation products in the $d b / d b$ mouse. Diabetes 51: 2082-2089

43. Vlassara H, Cai W, Crandall J et al (2002) Inflammatory mediators are induced by dietary glycotoxins, a major risk factor for diabetic angiopathy. Proc Natl Acad Sci USA 99: 15596-15601

44. Wolf M, Sandler L, Hsu K, Vossen-Smirnakis K, Ecker JL, Thadhani R (2003) First-trimester C-reactive protein and subsequent gestational diabetes. Diabetes Care 26:819-824 
45. Bo S, Signorile A, Menato G et al (2005) C-reactive protein and tumor necrosis factor- $\alpha$ in gestational hyperglycemia. J Endocrinol Invest. 28:779-786

46. Kankova K, Sebekova K (2005) Genetic variability in the RAGE gene: possible implications for nutrigenetics, nutrigenomics, and understanding the susceptibility to diabetic complications. Mol Nutr Food Res 49:700-709

47. Jiang R, Ma J, Ascherio A, Stampfer MJ, Willett WC, Hu FB (2004) Dietary iron intake and blood donations in relation to risk of type 2 diabetes in men: a prospective cohort study. Am J Clin Nutr 79:70-75

48. Lao TT, Ho LF (2004) Impact of iron deficiency anemia on prevalence of gestational diabetes mellitus. Diabetes Care 27:650-656

49. Cuco G, Fernandez-Ballart J, Sala J et al (2006) Dietary patterns and associated lifestyles in preconception, pregnancy and postpartum. Eur J Clin Nutr 60:364-371

50. Scholl TO (2005) Iron status during pregnancy: setting the stage for mother and infant. Am J Clin Nutr 81:1218S-1222S 